\title{
Optically Tunable Seebeck Effect from Intramolecular Proton-Transfer Materials in Organic Vertical Thin-Film Thermoelectric Device
}

Dehua Hu, ${ }^{1,5}$ Qing Liu, ${ }^{1}$ Jeremy Tisdale, ${ }^{1}$ Haerim Nam, ${ }^{2}$ Soo Young Park, ${ }^{2}$ Hsin Wang $^{3}$, Augustine Urbas, ${ }^{4}$ and $\mathrm{Bin}^{\mathrm{H}} \mathrm{u}^{1}{ }^{*}$

${ }^{1}$ Department of Materials Science and Engineering, University of Tennessee, Knoxville, TN 37996, USA

${ }^{2}$ Department of Materials Science and Engineering, ENG445, Seoul National University San 56-1, Shillim-dong, Kwanak-gu, Seoul 151-744, Korea

${ }^{3}$ Oak Ridge National Laboratory, Oak Ridge, TN, 37831, USA

${ }^{4}$ Air Force Research Laboratory, Wright Patterson Air Force Base, OH 45433, USA

${ }^{5}$ Institute of Polymer Optoelectronic Materials and Devices, State Key Laboratory of Luminescent Materials and Devices, South China University of Technology, Guangzhou 510640, P. R. China.

Correspondence to: Bin Hu (E-mail: $\underline{\text { bhu@utk.edu })}$

Keywords: Seebeck effect; proton-transfer; electron-phonon coupling; organic semico nductors. 
Abstract: This paper reports Seebeck effects from optically-induced intramolecular proton-transfer HPI-Cbz molecules based on vertical electrode/organic film/electrode thin-film devices. We observed large Seebeck coefficients of $428 \mu \mathrm{V} / \mathrm{K}$ and $390 \mu \mathrm{V} / \mathrm{K}$ from HPI-Cbz based thin-film devices at $60{ }^{\circ} \mathrm{C}$ when proton-transfer was induced by the photoexcitation of a $325 \mathrm{~nm}$ laser with an intensity of $12 \mathrm{~mW} / \mathrm{cm}^{2}$ and $6 \mathrm{~mW} / \mathrm{cm}^{2}$ respectively. Under dark condition without proton transfer occurring, the Seebeck coefficient was measured to be $342 \mu \mathrm{V} / \mathrm{K}$ at $60{ }^{\circ} \mathrm{C}$. The Seebeck coefficient enhancement by the induced intramolecular charge transfer can be attributed to the enhanced polarization difference between high- and low-temperature surface due to the stronger electron-phonon coupling followed with the proton-transfer in HPI-Cbz under photoexcitation, and the strength of electron-phonon coupling is proportional to the photoexcitation intensity. The enhanced temperature-dependent electrical polarization between the high and low-temperature surfaces acts as an additional driving force to diffuse the majority charge carriers for the development of a large Seebeck effect. Therefore, using intramolecular proton-transfer presents an effective approach of enhancing Seebeck effect in organic materials. 


\section{Introduction}

The Seebeck coefficient, $S$, is a critical parameter to increase the efficiency of the thermoelectric energy conversion and the value of materials' dimensionless figure of merit, $Z T$. The Seebeck coefficient is calculated by $S=\frac{\Delta V}{\Delta T}$, where $\Delta V$ and $\Delta T$ are the electrical potential difference and temperature difference across a medium between high and low temperature surfaces. The figure of merit is given by $Z T=\left(S^{2} \sigma / \kappa\right) T$, where $S, \sigma, T$, and $\kappa$ are the Seebeck coefficient, electrical conductivity, absolute temperature, and thermal conductivity, respectively. ${ }^{[1-5]}$ Generally, the Seebeck effect originates from charge (electrons or holes) diffusion, determined by the electronic structures and the scattering of charge carriers, between high and low-temperature surfaces in a material. ${ }^{[6-8]}$ High and low-temperature surfaces can respectively exhibit higher and lower densities of energetic carriers due to thermal activation, establishing an entropy difference in carrier concentration between the high and low-temperature surfaces. This entropy difference acts as the basic driving force to diffuse the majority carriers from high to low-temperature surface for the development of Seebeck effects. In order to get a high figure of merit $(Z T)$, increasing electrical conductivity of the materials is usually considered. However, a high electrical conductivity usually leads to a smaller Seebeck coefficient because increasing charge density causes a decreased traditional driving force of entropy difference across a given section and an increased electronic contribution to the thermal conductivity, both of which are undesirable for thermoelectric applications. ${ }^{[9-15]}$ Therefore, it is necessary to find an additional driving 
force for the charge diffusion to develop the Seebeck effect. In this paper, we propose to utilize the difference of surface polarization as the additional driving force for the carrier diffusion by constructing the thermoelectric device with a vertical multi-layer ITO/organic-thin-film/Au architecture. The photo-induced intramolecular proton-transfer material, HPI-Cbz, ${ }^{[16]}$ is sandwiched between two electrodes, creating organic/inorganic interfaces at the high- and low- temperature sides for the development of surface polarization. This surface polarization exhibits temperature-dependent and optically-tunable characteristics. First, the temperature-dependent thermal vibration of the organic molecule can lead to the temperature-dependent surface polarization difference between high and low-temperature surfaces through the electron-phonon coupling mechanism. ${ }^{[17-23]}$ This surface polarization difference produces a temperature-dependent built-in electrical field across the polymer film between high and low-temperature surfaces, which acts as an additional driving force for the development of the Seebeck effect. Second, because the charge density is proportional to the photoexcitation intensity before the saturation of excited states, ${ }^{[24]}$ the electron-phonon coupling strength can be optically tuned by changing the photoexcitation intensity, and the surface electrical polarization difference is then optically tunable. As a result, this hybrid thermoelectric device exhibits optically tunable enhanced Seebeck effects.

\section{Material and methods}

The ESIPT material HPI-Cbz was synthesized following the previous literature. ${ }^{[16]}$ The hybrid thin-film thermoelectric devices were fabricated with a sandwich 
architecture of ITO/HPI-Cbz/Au. The HPI-Cbz film was deposited on the cleaned ITO in a vacuum with a thickness of about $200 \mathrm{~nm}$. Then a top metal layer of Au was deposited on the organic film with a thickness of about $60 \mathrm{~nm}$ in a vacuum of $2 \times 10^{-6}$ Torr. K-type thermocouple wires were connected to the ITO and top metal layers to measure the temperature of each layer. The temperature difference was controlled by a high temperature-probe station system. During the Seebeck measurement, an extra current injected ITO plane was used to heat ITO-side of the device, now referred to as the hot side, through the glass substrate. At the same time an aluminum cube was placed close to the top metal layer, now referred to as the cold side, as a heat sink to generate a temperature difference. The $325 \mathrm{~nm}$ photoexcitation $\left(12 \mathrm{~mW} / \mathrm{cm}^{2}\right)$ from an IK series He-Cd laser was used to generate excited states from the transparent ITO hot side. All voltage and temperature data were collected by a Labview data acquisition system from Lucas. The temperature and electrical potential differences between high and low temperature surfaces are given by $\Delta T=T_{\text {hot }}-T_{\text {cold }}, \Delta V=V_{\text {cold }}-V_{\text {hot }}-V_{\text {oc. }}$ The Seebeck coefficients are calculated by $S=\frac{\Delta V}{\Delta T}$. The temperature difference $\Delta T$ of about $5 \mathrm{~K}$ was used in all the measurements. The electrical potential difference between high and low temperature surfaces $\Delta V$ was observed at values from several hundreds of $\mu \mathrm{V}$ to over one-thousand $\mu \mathrm{V}$. The accuracies for $\Delta V$ and $\Delta T$ are about 10 $\mu \mathrm{V}$ and $0.1 \mathrm{~K}$, respectively. It should be noted that both the photovoltaic effect and the Seebeck effect produce electric potential differences when photoexcitation and temperature difference are simultaneously applied. A Seebeck voltage can be developed when an entropy difference drives the majority carriers to diffuse into the 
polymer film from hot (ITO) side to cold $(\mathrm{Au})$ side, generating an electrical potential.

On the other hand, a photovoltaic voltage can be generated due to the dissociation of photoexcitation-generated excited states, leading to an electrical potential difference, similar to the open-circuit voltage $\left(V_{\mathrm{oc}}\right)$ in organic solar cells, with the positive $\mathrm{Au}$ electrode and the negative ITO electrode. Here, we used the following procedures to separate the Seebeck effect from the photovoltaic effect. First, the total electrical potential containing the Seebeck and the photovoltaic components was measured under a temperature difference of around $3{ }^{\circ} \mathrm{C}$ at different temperatures from $30{ }^{\circ} \mathrm{C}$ to $60{ }^{\circ} \mathrm{C}$ with constant photoexcitation intensity, denoted as $V_{\text {all }}$. Second, the photovoltaic voltage $\left(V_{\text {oc }}\right)$ was recorded immediately with a negligible temperature difference $\left(\Delta \mathrm{T}<0.1{ }^{\circ} \mathrm{C}\right)$ at different temperatures when the photoexcitation was applied. Third, the Seebeck voltage data $\left(V_{\mathrm{TE}}\right)$ was collected, as determined by the difference between the total electrical voltages and the photovoltaic voltages using the formula, $V_{\mathrm{TE}}=V_{\mathrm{all}}-V_{\mathrm{oc}}$. Finally, the Seebeck voltage data was used to calculate the Seebeck coefficients for the ITO/polymer/Au device at different temperatures. The capacitance measurements were performed on the fabricated devices, using an Agilent E4980A LCR meter with zero DC bias and $50 \mathrm{mV}$ AC field, to explore surface polarizations at different temperatures.

\section{Results and discussion}

Figure 1(a) shows the Seebeck coefficients generated from the multi-layer ITO/HPI-Cbz/Au device with the HPI-Cbz film thickness of about $200 \mathrm{~nm}$ at various temperatures. In the dark condition, the Seebeck coefficient $(S)$ of the device based on 
the HPI-Cbz films is positive and exceeds $342 \mu \mathrm{V} / \mathrm{K}$ at $60{ }^{\circ} \mathrm{C}$. Under photoexcitation of $6 \mathrm{~mW} / \mathrm{cm}^{2}$ and $12 \mathrm{~mW} / \mathrm{cm}^{2}$ at $325 \mathrm{~nm}$, the Seebeck coefficient of the ITO/HPI-Cbz/Au device increases throughout the whole measured temperature range compared with that in dark condition, reaching $380 \mu \mathrm{V} / \mathrm{K}$ and $428 \mu \mathrm{V} / \mathrm{K}$ at $60{ }^{\circ} \mathrm{C}$, respectively. This behavior is explained by the surface polarization difference that contributes to the development of the Seebeck effect. It is well known that the organic semiconducting materials usually exhibit strong coupling between charge carries and thermal vibrations due to coulombic interactions between delocalized $\pi$ electrons and thermal vibrations in a low dielectric background. This strong coupling between charge carries and temperature-dependent thermal vibrations will lead to a temperature-dependent surface polarization, generating a polarization difference between the high and low- temperature surfaces. This polarization difference between high and low- temperature surfaces, in addition to entropy difference under the temperature difference, drives the diffusion of positive charge carriers from the hot side to the cold side, generating a Seebeck voltage. In the excited state, the molecules demonstrate large dipole moments accompanied by significant changes of the configuration coordinate from ground states to excited states due to the redistribution of charge carriers, resulting in enhanced electron-phonon coupling strength. ${ }^{[25,26]}$ This enhanced electron-phonon coupling strength in excited states magnifies the driving force of electrical polarization difference between the high and low- temperature surfaces, and consequently enhances the Seebeck effect in excited states. Increasing photoexcitation intensity leads to stronger electron-phonon coupling, which 
accordingly enlarges the driving force of polarizatrion difference between high and low-temperature surfaces. Therefore, the Seebeck coefficient with photoexcitation intensity of $12 \mathrm{~mW} / \mathrm{cm}^{2}$ is larger than that with photoexcitation intensity of 6 $\mathrm{mW} / \mathrm{cm}^{2}$.

Here, it should be noted that photoexcitation can also lead to a photovoltage in the ITO/HPI-Cbz/Au device in the measurements. Here, we reveal the effective Seebeck effect generated at excited states with forward, reverse, and negligible temperature difference under photoexcitation, and we obtain the effective Seebeck voltage by subtracting the photovoltaic voltage from the total voltage measured with the device. As shown in Figure 1(b), the total voltage measured with forward temperature difference $(\mathrm{ITO}(\mathrm{hot}) / \mathrm{HPI}-\mathrm{Cbz} / \mathrm{Au}(\mathrm{cold}))$ under photoexcitation demonstrates larger values than the photovoltaic voltage measured with negligible temperature difference under photoexcitation. This indicates the same polarity of the photovoltaic and Seebeck voltages with the forward temperature difference. While applying a reverse temperature difference (ITO(cold)/HPI-Cbz/Au(hot)), the measured total electrical voltage of the device under photoexcitation is samller than the photovoltaic voltage measured with negligible temperature difference under photoexcitation, indicating opposite polarities of the photovoltaic and Seebeck voltages with the reverse temperature difference. Therefore, the difference of the entire electrical voltage between forward, negligible, and reverse temperature differences confirms that our data processing of subtracting the photovoltaic voltage from the total electrical voltage can determine the Seebeck voltage in the ITO/HPI-Cbz/Au device under 
photoexcitation and temperature difference.

Temperature-dependent photoluminescence spectra are used to study the charge-phonon coupling in the film. It has been reported that HPI-Cbz is an intramolecular proton transfer (ESIPT) material at excited states. ${ }^{[16]}$ As shown in Figure 2(a), the photophysics processes of HPI-Cbz undergo an intrinsic four-level cyclic proton-transfer reactions $\left(\mathrm{E} \rightarrow \mathrm{E}^{*} \rightarrow \mathrm{K}^{*} \rightarrow \mathrm{K} \rightarrow \mathrm{E}\right) .{ }^{[27]}$ The emission peaks around $390 \mathrm{~nm}$ and $462 \mathrm{~nm}$ are assigned to the E-emission and K-emission respectively. As shown in Figure 2(b), clearly, the peak intensity ratio of E-emission/K-emission increases with increasing temperature, and this reflects the enhanced coupling strength between the electronic transitions and thermal vibrations. As temperature increases, more intensive molecular vibrations cause significant deformation of molecular configuration. This significant deformation of molecular configuration would break the built-in intramolecular H-bond, which is essential for the efficient ESIPT reaction since the proton transfer reaction takes place on the locus of intramolecular H-bond. Therefore, more $\mathrm{E}$ form is obtained with increasing temperature, and the peak intensity ratio of E-emission/K-emission increases with increasing temperature. It should be noted that high temperature can increase the chance of non-radiative decay of both K-emission and E-emission. If the decrease of K-emission intensity is only attributed to the non-radiative decay at high temperature, there should be no K-form degrading to E-form as temperature increases, under which condition no additional E-form would be formed and the E-emission intensity would not increase. However, as shown in the insert of Figure 2(b), the E-emission intensity 
around $390 \mathrm{~nm}$ increases with increasing temperature. Thus, there must be additional E-form formed in the process with increasing temperature. Therefore, the high temperature surface of the device should have a higher degree of molecular configuration disorder and molecular bond distortion from stronger molecular thermal vibration to break built-in intramolecular H-bonds that is essential for the efficient ESIPT reaction. As a consequence, the high temperature surface possesses stronger charge-phonon coupling from more intense thermal vibrations and local polarization, which causes stronger surface polarization at the high temperature surface. Therefore, surface polarization difference is developed under the temperature difference within the device to drive the diffusion of photoexcited majority charge carriers toward the development of Seebeck effect.

Here, we show the photoexcitation-intensity tunable Seebeck effect by analyzing PL spectra of HPI-Cbz at different photoexcitation intensities. Figure 2(c) shows the normalized PL spectra of HPI-Cbz film under different photoexcitation intensities at $325 \mathrm{~nm}$. Obviously, the relative intensity of E-emission around $390 \mathrm{~nm}$ increases with increasing photoexcitation intensity, indicating that more E-form of HPI-Cbz is formed with increased photoexcitation intensity. This means that a higher degree of molecular configuration disorder and bond distortion is obtained under larger photoexcitation intensity, and the charge-phonon coupling increases with the increasing photoexcitation intensity. ${ }^{[28-30]}$ Therefore, increasing photoexcitation intensity should increase the surface polarization difference between the high and low- temperature surfaces, and the Seebeck effect is enhanced with increased 
photoexcitation intensity.

In order to experimentally validate the temperature-dependent surface polarization in our vertical thin-film thermoelectric device, we measured the capacitance of this device in excited states at different temperatures since capacitance reflects surface polarization. Figure 3(a) show the $C$ - $V$ characteristics for the ITO/ HPI-Cbz /Au device at different temperatures in excited state. We can see that the capacitance for the devices increases with increasing temperature, which indicates increasing surface polarization with increasing temperature. Figure 3(b) shows the temperature-dependent capacitance measurements performed on the fabricated devices at DC zero bias. The capacitance of the device at DC zero bias increases with increasing temperature from $30{ }^{\circ} \mathrm{C}$ to $60{ }^{\circ} \mathrm{C}$. This temperature-dependent capacitance result confirms that the electrical polarization difference between the high and low-temperature surfaces increases with increasing temperature. The high temperature surface possesses stronger electrical polarization compared to the low temperature surface.

The current-voltage characteristics are employed to investigate the electrical conductivities of the thermoelectric device. As shown in Figure 4, there is a significant increase in the current at excited states compared with the current in dark conditions for the HPI-Cbz film. Being calculated by the series resistance determined from the Cheung's function, ${ }^{[31]}$ the electrical conductivities are found to be $9.3 * 10^{-7}$ $\mathrm{S} / \mathrm{m}$ and $1.0^{*} 10^{-6} \mathrm{~S} / \mathrm{m}$ for photoexcitation intensities of $6 \mathrm{~mW} / \mathrm{cm}^{2}$ and $12 \mathrm{~mW} / \mathrm{cm}^{2}$, respectively, which are larger than that in dark condition of $6.6^{*} 10^{-7} \mathrm{~S} / \mathrm{m}$. The large 
enhancement of electrical conductivity can be explained by the photo-induced charge carriers as well as the lower band gap of the formed $\mathrm{K}^{*}$-tautomer in the excited state, which has a relative low energy band gap and facilitates charge transporting.

\section{Conclusions}

In summary, we present a new approach to optically tune the Seebeck effect of the organic film by using a hybrid vertical architecture of ITO/organic-thin-film/Au. On one hand, the coupling between local polarization and thermal vibration of the organic film leads to a polarization difference between the high and low temperature surface, which is increased in excited states. This polarization difference acts as a driving force to increase the Seebeck effect. On the other hand, as the electron-phonon coupling increases with the photoexcitation intensity, the temperature-dependent polarization difference is optically tunable, and consequently the Seebeck effect is optically tunable. Moreover, photo-induced free charges can increase electrical conductivity. Therefore, our experimental results demonstrate that the Seebeck effect as well as electrical conductivity can be optically tuned by using the vertical multi-layer electrode/organic materials/electrode devices. 


\section{Acknowledgements}

This research was supported by the financial supports from Air Force Office of Scientific Research (AFOSR) ( FA 9550-15-1-0064) and National Science Foundation (CBET-1438181). The authors also acknowledge the support from Sustainable Energy Education and Research Center and Center for Materials Processing at the University of Tennessee. This research was partially conducted at the Center for Nanophase Materials Sciences based on user project (CNMS2012-106, CNMS2012-107, CNMS-2012-108), which is sponsored at Oak Ridge National Laboratory by the Division of Scientific User Facilities, U.S. Department of Energy. The authors also acknowledge the supports from the National Significant Program (2014CB643506, 2013CB922104) and NSFC Program (61475051) in China. The authors also acknowledge the supports from National Science Foundation of China (Grant No. 51403063) and China Postdoctoral Science Fund (Grant No. 2014M562174).

\section{Author contributions}

D. H. performed the experiments and wrote the manuscript. Q. L. provided experimental assistance and was involved in the discussion and revision for the manuscript. J. T., H. W., and A. U. were involved in the discussion for the manuscript. H. N. and Y. P. provided the materials. B. H. directed the research project.

\section{Conflict of interest}

The authors declare no competing financial interests. Correspondence should be addressed to B. H. (bhu@utk.edu). 


\section{References}

[1] G-H. Kim, L. Shao, K. Zhang, K. P. Pipe, Nat. Mater. 12 (2013) 719.

[2] J. R. Sootsman, D. Y. Chung, M. G. Kanatzidis, Angew. Chem. Int. Ed. 48 (2009) 8616.

[3] M. S. Dresselhaus, G. Chen, M. Y. Tang, R. Yang, H. Lee, D. Wang, Z. Ren, J.-P. Fleurial, P. Gogna, Adv. Mater. 19 (2007) 1043.

[4] J. Yang, H.-L. Yip, A. K.-Y. Jen, Adv. Energy Mater. 13 (2013) 549.

[5] Q. Zhang, Y. Sun, W. Xu, D. Zhu, Adv. Mater. 2014, DOI: 10.1002/adma.201305371.

[6] J. P. Heremans, V. Jovovic, E. S. Toberer, A. Saramat, K. Kurosaki, A. Charoenphakdee, S. Yamanaka, G. J. Snyder, Science 321 (2008) 554.

[7] CRC Handbook of Thermoelectrics (Ed: D. M. Rowe), CRC Press, Boca Raton, FL, 1995.

[8] M. Cutler, N. F. Mott, Phys. Rev. 181 (1969) 1336.

[9] J. Ulańskia, J. K. Jeszkaa, M. Kryszewskia, Polym-Plast. Technol. Eng. 17 (1981) 139.

[10] B. Chandra, A. Afzali, N. Khare, M. M. El-Ashry, G. S. Tulevski, Chem. Mater. 22 (2010) 5179.

[11] I. Chung, J. H. Song, J. Im, J. Androulakis, C. D. Malliakas, H. Li, A. J. Freeman, J. T. Kenney, M. G. Kanatzidis, J. Am. Chem. Soc. 134 (2012) 8579.

[12] J. Sun, M. L.Yeh, B. J. Jung, B. Zhang, J. Feser, A. Majumdar, H. E. Katz, Macromolecules 43 (2010) 2897.

[13] T. Nakamura, K. J. Yashiro, K. Sato, J. Mizusaki, Mater. Chem. Phys. 122 (2010) 250.

[14] Y. Inoue, M. Kitano, K. Nakajima, M. Hara, Chem. Lett. 40 (2011) 410411.

[15] N. Mateeva, H. Niculescu, J. Schlenoff, L. R. Testardic, J. Appl. Phys, 83 (1998) 3111.

[16] S. Park, J. Seo, S. H. Kim, S. Y. Park, Adv. Funct. Mater. 18 (2008) 726.

[17] D. Hu, Q. Liu, J. Tisdale, T. Lei, J. Pei, H. Wang, A. Urbas, B. Hu, ACS Nano 9 (2015) 5208.

[18] Q. Liu, T. Wu, Y. Hsiao, M. Li, D. Hu, H. Wang, H. Xu, J. Tisdale, B. Hu, J. Mater. Chem. A (2015) DOI: 10.1039/C5TA02712H.

[19] J. Clark, C. Silva, R. Friend, F. Spano, Phys. Rev. Lett. 98 (2007) 206406.

[20] R. Hildner, U. Lemmer, U. Scherf, M. van Heel, J. Köhler, Adv. Mater. 19 (2007) 1978.

[21] E. G. Maksimov, G. P. Motulevich, Soviet Physics Jetp 34 (1972) 219.

[22] A. H. Castro Neto, F. Guinea, Phys. Rev. B 75 (2007) 045404.

[23] Q. Liu, D. Hu, H. Wang, M. Stanford, H. Wang, B. Hu, Phys. Chem. Chem. 
Phys. 16 (2014) 22201.

[24] A. J. Heeger, Chem. Soc. Rev. 39 (2010) 2354.

[25] P. J. Brown, D. S. Thomas, A. Köhler, J. S. Wilson, J.-S. Kim, C. M. Ramsdale, H. Sirringhaus, R. H. Friend, Phys. Rev. B 67 (2003) 064203.

[26] M. Pope, C. E. Swenberg, Electronic Processes in Organic Crystals and Polymers, 2nd ed., Oxford University Press, Oxford, 1999.

[27] J. E. Kwon, S. Y. Park, Adv. Mater. 23 (2011) 3615.

[28] N. Sai, Z. Q. Li, M. C. Martin, D. N. Basov, M. D. Ventra, Phys. Rev. B 75 (2007) 045307.

[29] S. R. Phillpot, Phys. Rev. B 35 (2007) 7533.

[30] F. C. Spano, J. Chem. Phys. 122 (2005) 234701.

[31] S. K. Cheung, N. W. Cheung, Appl. Phys. Lett. 49 (1986) 85. 

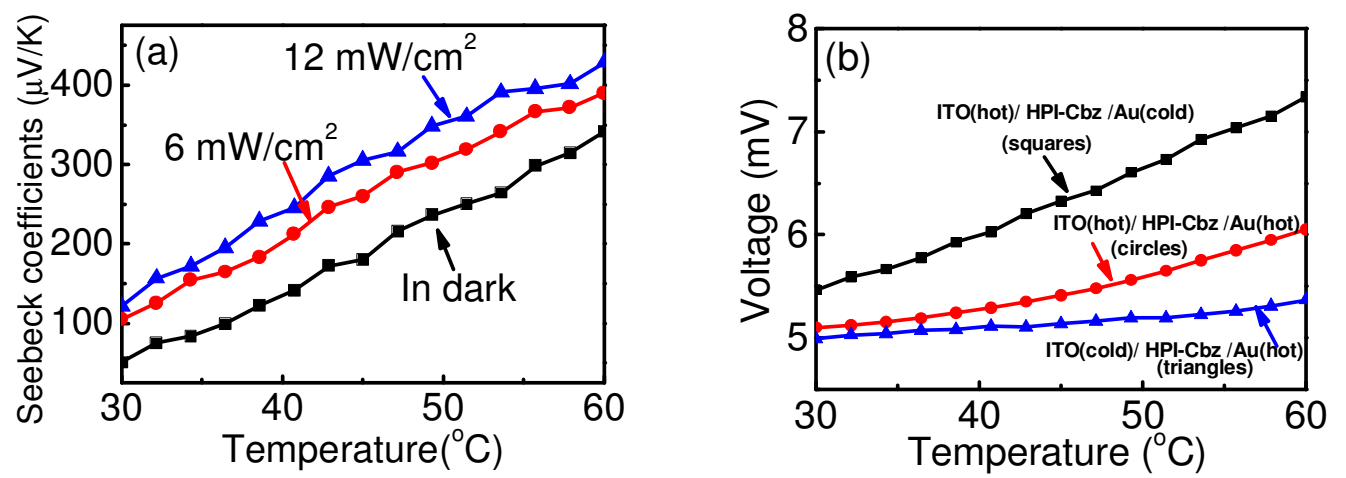

Figure 1. (a) Seebeck coefficients measured from ITO/HPI-Cbz/Au device in dark conditions and under photoexcitation of $12 \mathrm{~mW} / \mathrm{cm}^{2}$ and $6 \mathrm{~mW} / \mathrm{cm}^{2}$ at $325 \mathrm{~nm}$. (b) Overall voltages of the ITO/HPI-Cbz/Au device with forward, reverse and negligible temperature differences at different temperatures. 

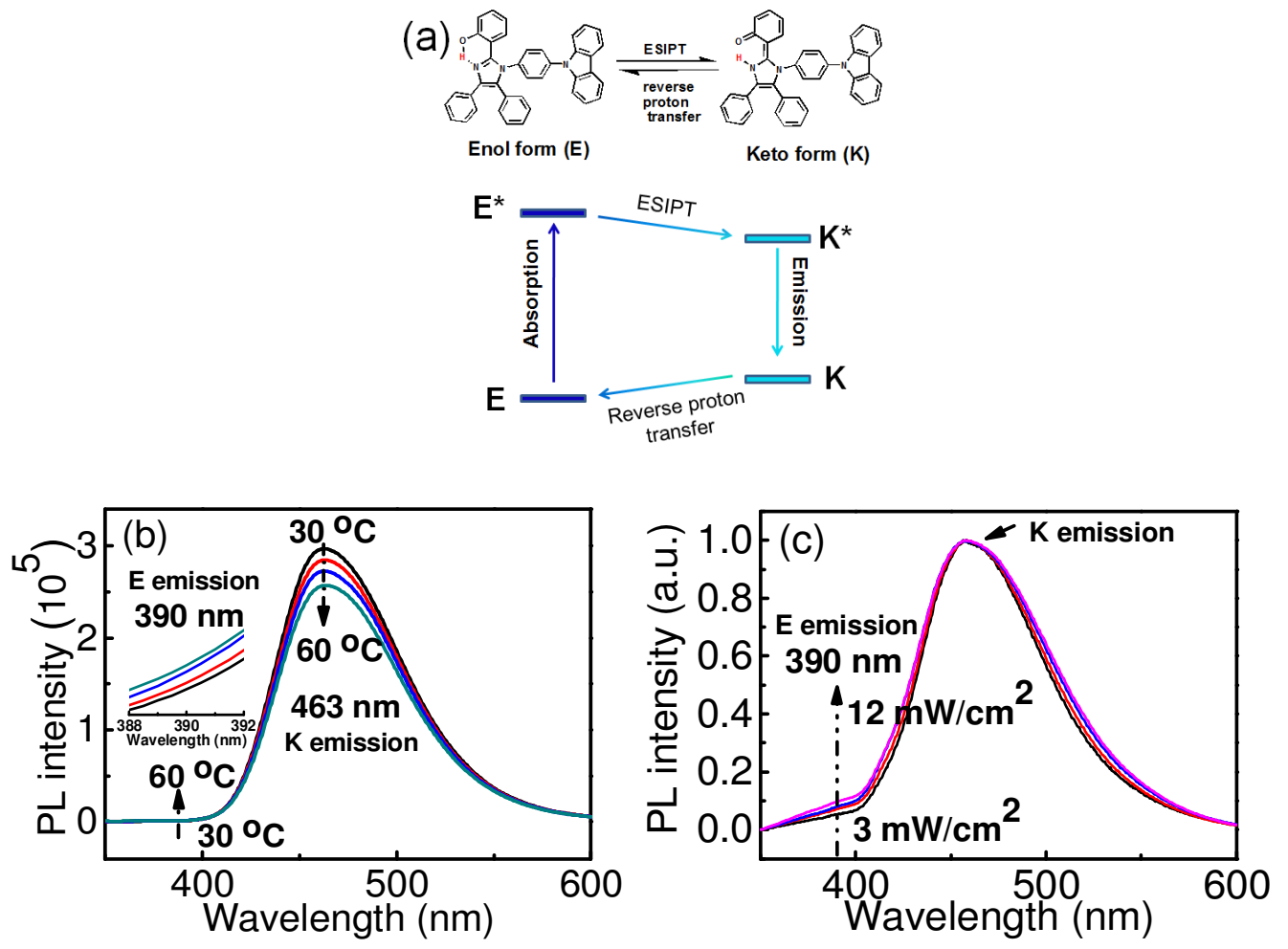

Figure 2. (a) Schematic representation of the ESIPT photocycle. (b) The PL spectra of HPI-Cbz in film at different temperature. (c) The normalized PL spectra of HPI-Cbz in film under different photoexcitation intensities. 

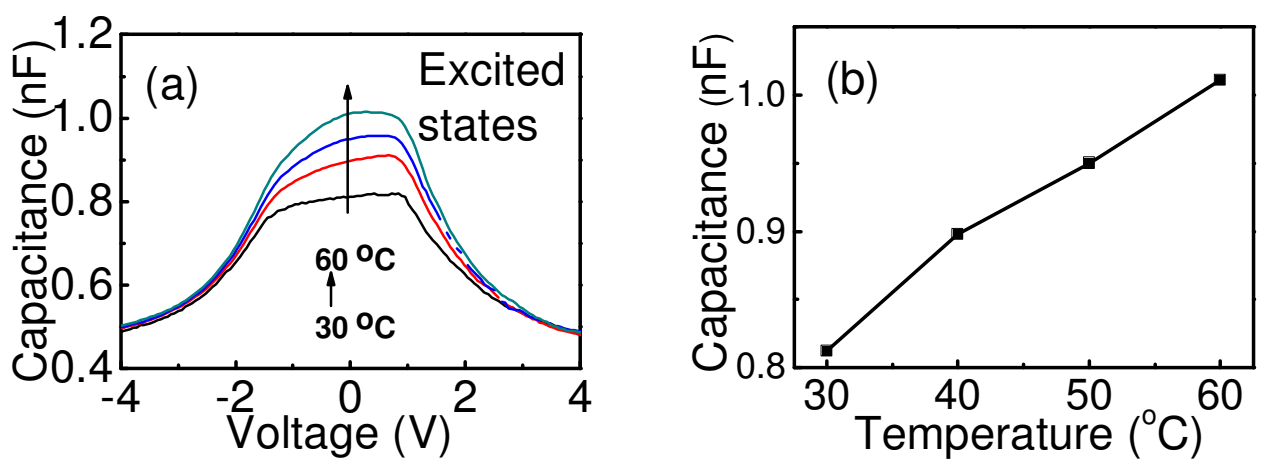

Figure 3. (a) The temperature-dependent capacitance characteristics of the ITO/HPI-Cbz/Au device after annealing at $100^{\circ} \mathrm{C}$ for $30 \mathrm{~min}$. (b) the temperature-dependent capacitance at DC zero bias in excited states with the device ITO/ HPI-Cbz/Au. 


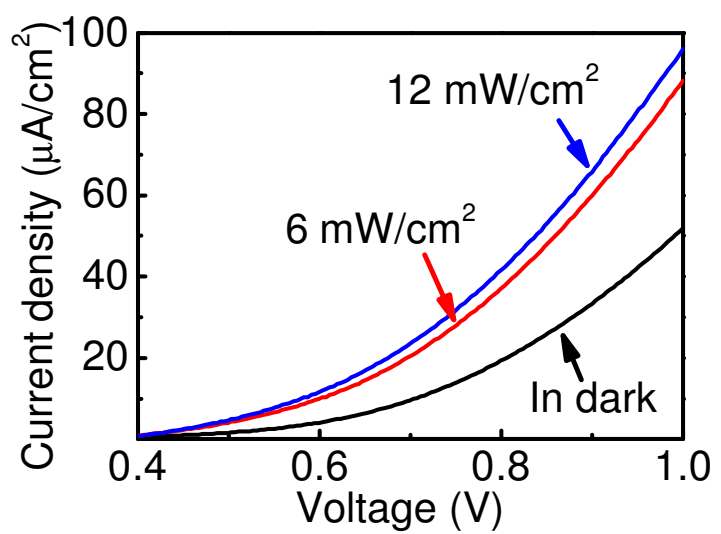

Figure 4. Current-voltage characteristics measured from ITO/HPI-Cbz/Au device in dark and under illumination. 


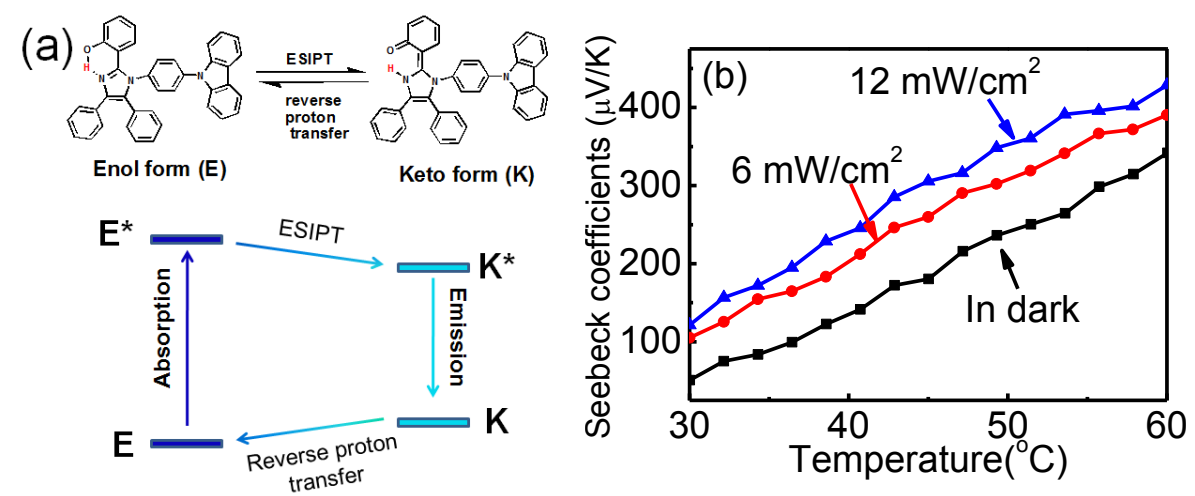

